\title{
Accelerometer-derived physical activity estimation in preschoolers - comparison of cut-point sets incorporating the vector magnitude vs the vertical axis
}

Claudia S. Leeger-Aschmann ${ }^{1}$, Einat A. Schmutz ${ }^{1}$, Annina E. Zysset ${ }^{2}$, Tanja H. Kakebeeke ${ }^{2,3}$, Nadine Messerli-Bürgy ${ }^{4,5}$, Kerstin Stülb ${ }^{6}$, Amar Arhab ${ }^{5}$, Andrea H. Meyer ${ }^{6,7}$, Simone Munsch ${ }^{6+}$, Oskar G. Jenni ${ }^{2,3 \dagger}$, Jardena J. Puder ${ }^{5+}$ and Susi Kriemler ${ }^{1 * \dagger}$

\begin{abstract}
Background: ActiGraph accelerometers are a widely used tool to objectively measure physical activity (PA) behavior in young children and several validated cut-point sets exist to estimate time spent in different PA intensities (sedentary time, light PA, moderate-to-vigorous PA). Applying different cut-point sets leads to large and meaningful differences in results. So far, only cut-point sets validated for the vertical axis have been compared and only the influence on time spent in moderate-to-vigorous PA has been analyzed.

Methods: A range of validated cut-point sets with their respective epoch length was applied to analyze cross-sectional data of the Swiss Preschoolers' Health Study (SPLASHY): 1) Vertical axis in combination with an epoch length of $15 \mathrm{~s}$ (VA-15), 2) Vertical axis in combination with an epoch length of $60 \mathrm{~s}$ (VA-60) and 3) Vector magnitude in combination with an epoch length of $60 \mathrm{~s}$ (VM-60). PA was measured for eight consecutive days using ActiGraph accelerometers (wGT3X-BT). Three days were required to be included in the analysis (minimum two weekdays and one weekend-day with at least ten hours recording per day).

Results: Four hundred forty-five preschoolers (mean age $3.9 \pm 0.5$ years; $46 \%$ girls) had valid accelerometer measurements. A longer epoch (VA-60 vs VA-15) resulted in 2\% less sedentary time (ST), 18\% more light PA (LPA) and 51\% less moderate-to-vigorous PA (MVPA); using the vector magnitude compared to the vertical axis (VM-60 vs VA-60) resulted in 34\% less ST, 27\% more LPA and 63\% more MVPA (all $p \leq 0.001$ ). Comparing all three sets of cut-points, ST ranged from 4.0 to $6.2 \mathrm{~h}$, LPA from 5.1 to $7.6 \mathrm{~h}$ and MVPA from 0.8 to $1.6 \mathrm{~h}$.
\end{abstract}

Conclusions: Estimated time spent in different PA intensities was strongly influenced by the choice of cut-point sets. Both, axis selection and epoch length need to be considered when comparing different studies especially when they relate PA behavior to health. The differences in the prevalence of children fulfilling PA guidelines highlight the relevance of these findings.

Trial registration: Current Controlled Trials ISRCTN41045021 (date of registration: 21.03.2014).

Keywords: Accelerometry, Behavior, Child, Methodology, Movement, SPLASHY

\footnotetext{
* Correspondence: susi.kriemlerwiget@uzh.ch

†'simone Munsch, Oskar G. Jenni, Jardena J. Puder and Susi Kriemler shared

last authors

${ }^{1}$ Epidemiology, Biostatistics and Prevention Institute, University of Zurich,

Hirschengraben 84, 8001 Zurich, Switzerland

Full list of author information is available at the end of the article
}

(c) The Author(s). 2019 Open Access This article is distributed under the terms of the Creative Commons Attribution 4.0 International License (http://creativecommons.org/licenses/by/4.0/) which permits unrestricted use, distribution, and reproduction in any medium, provided you give appropriate credit to the original author(s) and the source, provide a link to the Creative Commons license, and indicate if changes were made. The Creative Commons Public Domain Dedication waiver (http://creativecommons.org/publicdomain/zero/1.0/) applies to the data made available in this article, unless otherwise stated. 


\section{Background}

Achieving sufficient physical activity (PA) levels in the preschool age is not only important for the healthy development of children [1], but also for establishing movement habits that last throughout adolescence and adulthood [2]. To assess PA behavior in early childhood it is essential to have accurate and objective methods. A reliable and valid tool to objectively measure PA in preschoolers are ActiGraph accelerometers [3]. With these widely used devices the raw acceleration signal is collected at a pre-specified frequency and converted into counts per user-defined time period (epoch length). Age-specific activity thresholds (cut-points) are defined to distinguish sedentary time (ST) vs light PA (LPA) vs moderate-to-vigorous PA (MVPA) [3-7] and may therefore not be valid to assess time spent in certain PA intensities for other age groups due to different PA patterns [8]. These cut-points are validated for a certain combination of axes and epoch lengths. Movement can be measured either on the three-dimensional vector magnitude or only on the vertical axis (also known as axis 1), which is the most frequently used due to the previous lack of availability of the vector magnitude. The vector magnitude incorporates the vertical axis (up-down) as well as the longitudinal (forward-backward) and lateral (left-right) axes. Given the movement patterns characterized by short bouts, it has been argued that short epoch lengths, such as 15 [9], five [8] or even two seconds [10] should be used to accurately capture PA in this young age group.

Like for older children, there is a large variety of applied cut-point sets in studies analyzing PA behavior in preschoolers [8]. Janssen et al. [11] compared the classification accuracy of six ActiGraph cut-point sets (incorporating the vertical axis and different epoch length) in preschoolers and recommended using the Evenson et al. [6] cut-point to differentiate between ST and LPA and the Pate et al. [3] cut-point for LPA and MVPA. However, using the vertical axis alone may not be appropriate to assess preschoolers' PA as young children behave in an omnidirectional manner. Despite the fact that three-dimensional ActiGraph devices have been available since 2009, all validation studies except one [4] used only the vertical axis to determine a cut-point set for hip-worn ActiGraph accelerometers [3-7]. There are two other validation studies for three-dimensional ActiGraph cut-points in preschoolers [12, 13] but neither research groups were able to give a complete cut-point set to distinguish between all intensities (ST, LPA, MPA and VPA).

Currently, no gold standard method exists to quantify activity behavior and no agreement has been reached on the most appropriate cut-points for preschool-aged children [14]. The lack of a consensus leads to challenges when comparing and pooling study results, potentially leading to invalid conclusions on the basis of which policy makers define PA guidelines. Applying different cut-point sets is known to create large and significant differences in the estimated time spent in MVPA, ranging from 30 to $260 \mathrm{~min} /$ day [15-17], and fulfillment of PA guidelines [18]. However, these studies focused only on the time spent in MVPA and did not investigate the variability in ST or LPA among different cut-point sets. Furthermore, only cut-point sets using the vertical axis were compared and the influence of the vector magnitude was neglected. To address these methodological gaps the aim of this study was to quantify the influence of different cut-point sets on the estimation of time spent in different PA intensities, covering the whole range from ST to MVPA. For this, physical activity estimation in preschoolers was investigated and results of cut-point sets incorporating the vector magnitude vs the vertical axis was compared. As it is known that different factors may influence PA behavior [19, 20] sub-group analyses according to sex, age and weight status can be found in the Additional file 1.

\section{Methods}

Study design and participants

SPLASHY (Swiss Preschoolers' Health Study) is a prospective, multi-center cohort study including 555 two to six years old children within Switzerland (ISRCTN41045021). Due to logistical reasons the recruitment and testing of healthy preschoolers took place in childcare centers. $20 \%$ of the 639 contacted childcare centers showed first interest, one third of those had to be excluded (mainly due to too few participants), so the final cohort consisted of 84 randomly selected childcare centers from five cantons (Aargau, Bern, Fribourg, Vaud and Zurich) stratified for living area (urban vs rural) and socio-economic region (high vs low) [21]. To obtain a large external validity, exclusion criteria were kept at a minimum; all preschoolers, able to perform the testing (e.g. no motor or cognitive disability), were invited to participate in SPLASHY. The cantonal ethical committee of each study site approved the study protocol and the study was conducted in accordance with the Declaration of Helsinki. Parents gave their written informed consent for study participation and children consented orally.

\section{Measurements}

Anthropometric data were assessed during testing afternoons in the childcare centers. Standing height was measured to the nearest $0.5 \mathrm{~cm}$ using a measuring tape. Weight was measured to the nearest $0.1 \mathrm{~kg}$ using an electronic scale (Seca, Basel, Switzerland). BMI percentiles were calculated according to World Health Organisation 
criteria and divided into normal weight ( $<85$ th percentile) and overweight ( $\geq 85$ th percentile) [22]

PA was measured for a week with a tri-axial accelerometer (wGT3X-BT, ActiGraph, Pensacola, Florida, USA). The device was attached to the child's right hip and parents/caregivers received detailed instructions on the use of the activity monitor. They were instructed to wear the monitor during all activities including the night, except for swimming and showering. The accelerometer was programmed to record PA data at a sampling frequency of $30 \mathrm{~Hz}$. Raw data were downloaded using the ActiLife v6.11.4 Firmware v1.0.0, saved as csv-files and further processed by $\mathrm{R}$ software (version 3.1.0). For data preparation all non-wear times, defined as time periods of consecutive zero activity counts of 20 min or more in all three axes [9, 23], were excluded. To enable the categorization of the PA intensities according to validated cut-point sets with their specific epoch length, data were aggregated to two versions of expanded epoch lengths of 15 and $60 \mathrm{~s}$. Based on the aggregated counts, the PA intensities were determined using three cut-point sets validated in preschoolers, which differ in axis selection (vertical axis [VA] vs vector magnitude $[\mathrm{VM}]$ ) and epoch length (15s vs $60 \mathrm{~s}$ ): 1) VA-15, 2) VA-60, and 3) VM-60. The cut-points differentiating SB from LPA and LPA from MVPA were 25 and 420 counts per $15 \mathrm{~s}$ for VA-15 [11]. The respective cut-points for VA-60 were 240 and $2120 \mathrm{cpm}$ and for VM-60 they were 820 and $3908 \mathrm{cpm}$. [4]. Due to the lack of validation studies, VM-15 could not be included.

Children aged between three to five years with minimum monitoring of three days (including two weekdays and one weekend-day) with at least 10 hours recording were included in analysis [24, 25]. Only PA data recorded between $7 \mathrm{am}$ and $9 \mathrm{pm}$ were analyzed. SPLASHY had two assessment waves and the first valid PA assessment for each child was taken for analysis. The number of monitoring days, average wear time (h/day), average PA (avPA, cpm), and the average time spent in the different PA intensities (min/day), including sedentary time (ST), light PA (LPA), moderate PA (MPA), moderate-to-vigorous PA (MVPA), vigorous PA (VPA) and any PA (LMVPA = LPA + MVPA) were extracted. The percentage of children fulfilling two widely accepted PA guidelines was used to show the relevance of our findings; the relatively loose PA guideline requesting 180 min LMVPA per day [26-28] and the more stringent one requesting 60 min MVPA per day $[18,29]$.

\section{Statistical analysis}

Linear multilevel models were applied to compare I) differences of avPA on the three axes (vertical, longitudinal and lateral) and the vector magnitude between both epoch lengths (15 vs $60 \mathrm{~s}$ ) and II) differences of time spent in various PA intensities (ST, LPA, MPA, MVPA, VPA and LMVPA) when applying different cut-point sets: a) VA-15 vs VA-60, b) VA-60 vs VM-60 and c) VA-15 vs VM-60. Each multilevel model also included wear time (h/day) as a fixed effect and an intercept of the subject's childcare center as random effect to account for the clustered sampling approach used in this study. Each subject only contributed a single observation to this analysis. Because the models showed light heteroscedasticity (that is, the variance of the outcome increased with larger values of the predictor), we report model results using the so-called "sandwich" estimator of the variance-covariance matrix, which is a more robust estimator of the variance that the usual one. The variance-covariance estimates have been calculated with the $\mathrm{R}$ package 'clubSandwich' (option type $=$ "CR1S"). The significance level $\mathrm{p}$ was set at 0.05 and all models were visually checked for normally distributed residuals using q-q plots. Descriptive statistics for the entire sample are presented in the paper and exploratory subgroup analyses stratified by age ( $3-3.49$ years; $3.5-3.99$ years; 4-4.49 years; $4.5-5$ years), sex (boys; girls) and weight status (BMI $<85 \%$ percentile; $\mathrm{BMI} \geq 85 \%$ percentile [22]) can be found in the Additional file 1 .

\section{Results}

The final sample consisted of 445 preschoolers aged three to five years (mean age $3.9 \pm 0.5$ years; $54 \%$ male). Mean height was $102.5 \pm 5.3 \mathrm{~cm}$ and mean weight $16.8 \pm$ $2.2 \mathrm{~kg}$; 334 (75.1\%) children were categorized as normal weight and 100 (22.5\%) as overweight. Average monitoring included $6.0 \pm 1.1$ days of recordings and mean wear time was $12.8 \pm 0.6 \mathrm{~h} /$ day. Children with missing PA data did not differ significantly from those included in the analysis according to sex, BMI, living area (urban-rural) or socio-economic state.

\section{Average counts on different axis}

Table 1 shows that avPA varied substantially depending on the axis; avPA assessed by axis 2 (longitudinal, forward-backward) and axis 3 (lateral, left-right) showed

Table 1 Mean average physical activity (avPA in cpm) and standard deviation for single axes and the three-dimensional vector magnitude $\left(\mathrm{VM}=\sqrt{\left.(\operatorname{axis} 1)^{2}+(\operatorname{axis} 2)^{2}+(\operatorname{axis} 3)^{2}\right)}\right.$ according to different epoch lengths (15 vs 60 s)

\begin{tabular}{lll}
\hline Average PA [cpm] & $15 \mathrm{~s}$ & $60 \mathrm{~s}$ \\
\hline Axis 1 & $623 \pm 152$ & $622 \pm 151$ \\
Axis 2 & $800 \pm 162$ & $799 \pm 161$ \\
Axis 3 & $894 \pm 176$ & $892 \pm 175$ \\
Vector magnitude & $1446 \pm 281$ & $1409 \pm 276$
\end{tabular}

Axis 1 denotes the vertical axis (up-down), axis 2 the longitudinal axis (forward-backward) and axis 3 the lateral axis (left-right) 
higher values than the vertical axis (axis 1, up-down) and therefore had a bigger impact on the three-dimensional vector magnitude. Furthermore, avPA was marginally but significantly higher with the shorter compared to the longer epoch length ( 15 vs $60 \mathrm{~s}$ ), in all axes and for the vector magnitude (all $p \leq 0.001$ ). Subgroup analyses revealed that the pattern was very similar (see Additional file 1: Tables S2, S3 and S4).

\section{Comparison of cut-point sets}

Figure 1 shows individual and combined influences of varying epoch length and axis selection based on observed data. A longer epoch length (15 vs 60 s) led to $2 \%$ less ST, $18 \%$ more LPA and 51\% less MVPA. Taking the vector magnitude (vs vertical axis) led to $34 \%$ less ST, 27\% more LPA and 63\% more MVPA. The combined impact of using a longer vs shorter epoch and the vector magnitude instead of vertical axis led to 35\% less ST, 51\% more LPA and 17\% less MVPA. All PA intensities (ST, LPA, MPA, MVPA, VPA, and LMVPA) differed significantly $(p<0.001)$ between the different cut-point sets (VA-15 vs VA-60; VA-60 vs VM-60; VA-15 vs VM-60) except VPA between VA-60 and VM-60, and MPA between VA-15 and VM-60 (both $p>0.05$; see Additional file 1: Table S5). Subgroup analyses revealed that the pattern and extent of change was strikingly similar (see Additional file 1: Tables S6, S7, S8 and Figure S1 a-h).

The prevalence of children fulfilling PA guidelines was evaluated (average across all valid days): All children fulfilled the recommendation requesting $180 \mathrm{~min}$ LMVPA per day; however, when applying the recommendation requesting $60 \mathrm{~min}$ MVPA per day, the percentage of preschoolers fulfilling this guideline was strikingly variable ranging from $90 \%$ for VA-15, to $22 \%$ for VA-60 and $63 \%$ for VM-60 (Additional file 1: Table S5). Subgroup analyses revealed very similar results (see Additional file 1: Tables S6, S7 and S8).

\section{Discussion}

The analysis approach of accelerometer-derived data has a huge impact on the outcome. This study showed that avPA was strikingly higher on the longitudinal and lateral axis compared to the commonly used vertical axis, but the influence of shorter compared to longer epoch length was marginal. Furthermore, time spent at different PA intensities varied substantially depending on the accelerometer cut-point set applied (VA-15, VA-60 and VM-60). This variation challenges the accurate depiction of PA and should be taken into consideration when developing PA guidelines.

\section{Average counts on different axes}

For avPA, 28 and $43 \%$ higher values were detected on the longitudinal and lateral axes respectively, compared to the commonly used vertical axis (Tab. 1) suggesting that simply taking the vertical axis as representative may not be valid to represent the PA behavior of each child. In children aged five to nine years, Jimmy et al. [30] also found varying avPA values for the different axes, which were dependent on specific activities: Walking activities

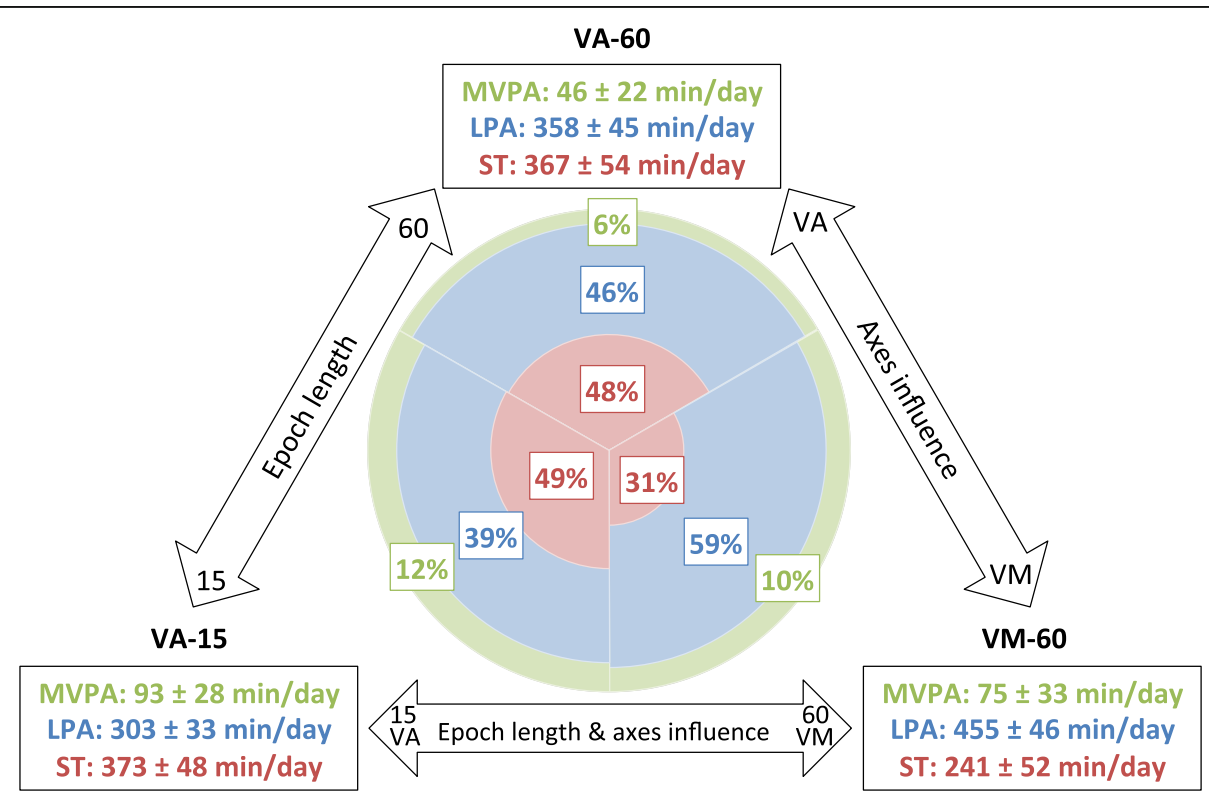

Fig. 1 Absolute values (mean \pm standard deviation) and percentages of time spent in different PA intensities (ST $=$ sedentary time, LPA $=$ light physical activity and MVPA = moderate-to-vigorous physical activity) according to three different cut-point sets: VA-15 denotes analysis using the vertical axis (VA) with a $15 \mathrm{~s}$ epoch length, VA-60 denotes analysis using the vertical axis (VA) with a $60 \mathrm{~s}$ epoch length and VM-60 denotes analysis using the vector magnitude (VM) with a $60 \mathrm{~s}$ epoch length 
and jogging resulted in the highest counts on the vertical axis but activities like playing with toy trains, free play and soccer led to more counts on the longitudinal and lateral axes than on the vertical axis. Our finding of varying activity counts among individual axes thus supports the use of the vector magnitude, which takes all axes of the three-dimensional system into account. Although avPA was significantly higher with shorter compared to longer epoch length, differences within axis were marginal and were therefore not clinically relevant. This was most likely due to the high co-linearity of the two variables and the integration over a longer interval, leading to smoothing of extreme values.

\section{Comparison of cut-point sets}

The results regarding time spent in different PA intensities differed tremendously depending on the applied cut-point set (Fig. 1): a) Effect of epoch lengths (VA-15 vs VA-60): A longer epoch length captured less MVPA because the behavior was classified as LPA; ST was only marginally influenced. Even though experts [8-10] postulate that shorter epoch lengths are better, preferably $15 \mathrm{~s}$ or less, due to preschoolers activity patterns in short bursts $[10,14]$, there is generally weak evidence to support this idea according to systematic reviews that correlated PA intensities and health in preschoolers [1]. Previous research showed that shorter epoch length capture more PA, despite converting the cut-points by dividing or multiplying to fit different epoch lengths; i.e. if the cut-point for $15 \mathrm{~s}$ epoch is 25 counts, it is 100 counts for an epoch length of $60 \mathrm{~s}$ [17, 31, 32]. However, several authors recommend using the same epoch length like during the validation study $[8,9,14]$. Additionally one should be aware that even reintegrating ActiGraph measurement with a short epoch length in longer epoch length results in more PA output compared to the recording with the respective longer epoch length [17]. b) Effect of axis selection (VA-60 vs VM-60): When using the vector magnitude (vs the vertical axis), children were categorized as being more active; they showed less ST, and more LPA and MVPA. This finding may not be surprising as the VM-60 cut-point set not only takes the movement on the vertical axis but also those on the longitudinal and lateral axes into account. A study in seniors showed similar results, as more LPA and MVPA was recorded when cut-points for the vector magnitude instead the vertical axis were applied [33]. Another study with children aged five to nine years concluded that their cut-points based on the vector magnitude did not appear to reflect the intensity categories more accurately than cut-points based on the vertical axis [34]. However, a very short epoch length of five seconds was used for calibration, which is known to be more sensitive to capturing high intensity activity than longer epoch lengths
[17]. c) Combined effect of epoch length and vector selection (VA-15 vs VM-60): The combined impact of a longer epoch and vector magnitude led to less ST, more LPA and less MVPA. Although we cannot resolve which cut-point set is more appropriate, from a behavioral perspective considering preschoolers' omnidirectional activity pattern in short bursts, the use of a three-dimensional system with a short epoch length makes logical sense to assess their PA behavior. Unfortunately, validation studies for this age group are still missing.

\section{General thoughts}

Our study is not the first comparing different validated ActiGraph cut-point sets and detecting the discrepancy in time spent in activity levels in preschoolers [14-17] and school-aged children [31, 35, 36]. Novel is that we looked at the effect of preschoolers' omni-directional movement behavior (e.g. vector magnitude vs single vertical axis) and focused on the whole range of PA behavior (ST, LPA and MVPA) rather than only at MVPA. The use of a wide variety of cut-point sets generates disparity in PA estimates leading to lack in comparability [15-17]. As a solution to this cut-point non-equivalence, some authors developed formulas, which convert PA estimates from one set of cut-points into estimates from another set of cut-point [37, 38]. Although these conversion formulas may facilitate comparisons across studies, they do not answer the question of which cut-points are most appropriate for the preschool population. Like previous authors [14, 35] we request a consensus about a common approach to analyze PA behavior by accelerometers. This will only be possible through additional series of calibration and independent validation studies. If we stay in this conventional system using company-based software for analyses, priorities should be given to validation of cut-point sets combining a short epoch length of $15 \mathrm{~s}$ or less with the three-dimensional VM, as this combination best reflect the natural PA behavior of preschoolers. Alternatively, we may agree on moving back to the use and documentation of raw acceleration signals, rather than proprietary counts, as proposed by a recent critical and elegant paper [39].

The ability to accurately estimate PA of young children is necessary to make well-informed decisions and potential recommendations for public health policies. The proportion of children engaging in the recommended 60 min of MVPA per day, that ranged from 22 to $90 \%$ depending on the set of cut-points applied, reflects the relevance of this statement. Despite ample evidence that adult diseases have their origins in childhood [40], evidence on the link between preschool PA and health outcomes is scarce [1]. Obviously preschoolers are generally healthy and non-communicable diseases develop much later and over decades, making the link between PA and 
health outcomes at this young age a true challenge. A better understanding of the amount, frequency and intensity of young children's PA for persistent health benefits is needed, as established for older children [41, 42]. This can only be reached by focusing on long-term cohorts that are able to relate PA behavior at preschool age with relevant health outcomes later in life. In the meantime comparability of study results can be reached by reporting the cut-point set independent avPA (in $\mathrm{cpm}$ ) and using conversion formulas as a tool to compare the PA behavior among studies.

\section{Strengths and limitations}

A strength of our study is the relatively large and randomly selected sample of preschoolers with a reliable and objective PA assessment. Furthermore, cut-point sets covering the whole range of PA intensities and validated for the newest ActiGraph generation (GT3X) in very similar age groups were studied. The chosen cut-point sets varied not only according to the epoch length but also axis used, showing the additional effect of measurement dimensions. Limitations of our study include that the study participation was voluntary and focused on children attending childcare centers, which may have led to a potential participation bias. Our analysis approach included a number of data selection decisions (number of days, length of day, and definition of non-wear time) and any of these decisions could have influenced the results. There are several constraints concerning measurement of PA by accelerometers such as imprecise assessments of rolling activities like riding bogie wheels and the incapability to measure water activities, which both could have led to an underestimation of PA. Even though nighttime sleep between $9 \mathrm{pm}$ and 7 am was removed, most of the preschoolers still took afternoon naps; this daytime sleep could have been measured incorrectly. Yet, all these limitations were true for all different versions of analyses, therefore any bias is expected to be equal among all applied cut-point sets.

\section{Conclusions}

The analysis of objectively assessed PA behavior of preschoolers is influenced by various factors. I) Average PA counts were strikingly higher on the longitudinal and lateral axis compared to the generally used vertical axis. This supports the use of the vector magnitude that takes all axes of the three-dimensional system into account. II) The choice of accelerometer cut-point set had a substantial impact on measured time spent in different PA intensities. Both, the epoch length and the choice of axis have to be considered when comparing different studies and may explain part of the differences in observed PA behavior. More validation studies that best reflect PA behavior of preschoolers (three-dimensional VM and short epoch length) are required. Additionally, more long-term research, able to relate PA behavior of preschoolers to health outcomes in later life, is needed. Meanwhile it is important to report not only the time spent in certain activity levels but also the cut-point independent avPA (in cpm) or raw acceleration signals to analyze amount and intensity of PA behavior to improve comparability between study results.

\section{Additional file}

\begin{abstract}
Additional file 1: Additional tables and subgroup analyses according to age, sex and weight status are summarized in the additional file of "Accelerometer-derived physical activity estimation in preschoolers -

Comparison of cut-point sets incorporating the vector magnitude vs the vertical axis". (PDF $592 \mathrm{~kb}$ )
\end{abstract}

\section{Abbreviations}

avPA: Average physical activity [cpm]; BMI: Body mass index; cpm: Count per minute; h/day: Hours per day; LMVPA: Any physical activity (LMVPA = LPA + MVPA) [min/day]; LPA: Light physical activity [min/day]; min/day: Minutes per day; MPA: Moderate physical activity [min/day]; MVPA: Moderate-to-vigorous physical activity [min/day]; PA: Physical activity; SPLASHY: Swiss Preschoolers' Health Study; ST: Sedentary time [min/day]; VA: Vertical axis; VM: Vector magnitude; VPA: Vigorous physical activity [min/day]

\section{Acknowledgements}

We would like to thank all children, families and day care centers that contributed data to SPLASHY. We also thank all students and the research team for their valuable contribution.

\section{Funding}

The study was funded through a Sinergia grant of the Swiss National Science Foundation (CRSII3_147673) and by the Jacobs Foundation. Both Foundations neither interfered with the design of the study nor provided any direct support (e.g., subject recruitment, data collection, analysis and interpretation of data or in writing the manuscript).

\section{Availability of data and materials}

The dataset supporting the conclusions of this article is available upon reasonable request to the corresponding author.

\section{Authors' contributions}

JJP, SK, SM, OGJ conceived and designed the SPLASHY study. AM was the responsible study statistician. CLA, EAS, AEZ, THK, KS, NMB, AA performed data collection. CLA performed data analysis and wrote, reviewed, and edited the manuscript. All authors reviewed, edited and approved the manuscript.

\section{Ethics approval and consent to participate}

The cantonal ethical committees of each study site approved the study protocol (Kantonale Ethikkommison Bern (KEK BE 070/13), Ethikkommission Nordwest- und Zentralschweiz (EKNZ 2013/083), Kantonale Ethikkommision Zürich (KEK ZH 2013-0568) and the main department in Lausanne Commission cantonale d'éthique de la recherche sur l'être humain (CER VD 338/13)) and the study was conducted in accordance with the Declaration of Helsinki. Parents gave their written informed consent for study participation and children consented orally.

Consent for publication

Not applicable

Competing interests

The authors declare that they have no competing interests. 


\section{Publisher's Note}

Springer Nature remains neutral with regard to jurisdictional claims in published maps and institutional affiliations.

\section{Author details \\ ${ }^{1}$ Epidemiology, Biostatistics and Prevention Institute, University of Zurich, Hirschengraben 84, 8001 Zurich, Switzerland. ${ }^{2}$ Child Development Center, University Children's Hospital Zurich, Steinwiesstrasse 75, 8032 Zurich, Switzerland. ${ }^{3}$ Children's Research Center, University Children's Hospital Zurich, Steinwiesstrasse 75, 8032 Zurich, Switzerland. ${ }^{4}$ Clinical Child Psychology \& Biological Psychology, University of Fribourg, Rue P.A. de Faucigny 2, 1700 Fribourg, Switzerland. ${ }^{5}$ Obstetric service, Department Woman-Mother-Child, Lausanne University Hospital, Avenue Pierre Decker 2, 1011 Lausanne, Switzerland. ${ }^{6}$ Department of Clinical Psychology and Psychotherapy, University of Fribourg, Rue P.A. de Faucigny 2, 1700 Fribourg, Switzerland. ${ }^{7}$ Department of Psychology, Division of Clinical Psychology and Epidemiology, University of Basel, Missionsstrasse 62A, 4055 Basel, Switzerland.}

\section{Received: 26 March 2018 Accepted: 15 April 2019} Published online: 06 May 2019

\section{References}

1. Timmons BW, Leblanc AG, Carson V, Connor Gorber S, Dillman C, Janssen I, Kho ME, Spence JC, Stearns JA, Tremblay MS. Systematic review of physical activity and health in the early years (aged 0-4 years). Appl Physiol Nutr Metab. 2012;37:4.

2. Twisk JW, Kemper HC, van Mechelen W. Tracking of activity and fitness and the relationship with cardiovascular disease risk factors. Med Sci Sports Exerc. 2000;32:8.

3. Pate RR, Almeida MJ, Mclver KL, Pfeiffer KA, Dowda M. Validation and calibration of an accelerometer in preschool children. Obesity (Silver Spring). 2006;14:11.

4. Butte NF, Wong WW, Lee JS, Adolph AL, Puyau MR, Zakeri IF. Prediction of energy expenditure and physical activity in preschoolers. Med Sci Sports Exerc. 2014;46:6.

5. van Cauwenberghe E, Labarque V, Trost SG, de Bourdeaudhuij I, Cardon G. Calibration and comparison of accelerometer cut points in preschool children. Int J Pediatr Obes. 2011:6:2-2.

6. Evenson KR, Catellier DJ, Gill K, Ondrak KS, McMurray RG. Calibration of two objective measures of physical activity for children. J Sports Sci. 2008;26:14

7. Sirard J, Trost S, Pfeiffer K, Dowda M, RR P. Calibration and evaluation of an objective measure of physical activity in preschool children. J Phys Act Health. 2005;3:345-57.

8. Migueles $\mathrm{JH}$, Cadenas-Sanchez C, Ekelund U, Delisle Nystrom C, MoraGonzalez J, Lof M, Labayen I, Ruiz JR, Ortega FB. Accelerometer data collection and processing criteria to assess physical activity and other outcomes: a systematic review and practical considerations. Sports Med. 2017; 47(9):1821-45

9. Cliff DP, Reilly JJ, Okely AD. Methodological considerations in using accelerometers to assess habitual physical activity in children aged 0-5 years. J Sci Med Sport. 2009;12:5.

10. Baquet G, Stratton G, Van Praagh E, Berthoin S. Improving physical activity assessment in prepubertal children with high-frequency accelerometry monitoring: a methodological issue. Prev Med. 2007;44:2.

11. Janssen X, Cliff DP, Reilly JJ, Hinkley T, Jones RA, Batterham M, Ekelund U, Brage S, Okely AD. Predictive validity and classification accuracy of ActiGraph energy expenditure equations and cut-points in young children. PLoS One. 2013;8:11

12. Costa S, Barber SE, Cameron N, Clemes SA. Calibration and validation of the ActiGraph GT3X+ in 2-3 year olds. J Sci Med Sport. 2014;17:6.

13. Pulakka A, Cheung YB, Ashorn U, Penpraze V, Maleta K, Phuka JC, Ashorn P. Feasibility and validity of the ActiGraph GT3X accelerometer in measuring physical activity of Malawian toddlers. Acta Paediatr. 2013;102:12.

14. Kim Y, Beets MW, Welk GJ. Everything you wanted to know about selecting the "right" Actigraph accelerometer cut-points for youth, but: a systematic review. J Sci Med Sport. 2012;15:4.

15. Bornstein DB, Beets MW, Byun W, Mclver K. Accelerometer-derived physical activity levels of preschoolers: a meta-analysis. J Sci Med Sport. 2011;14:6.
16. Cliff DP, Okely AD. Comparison of two sets of accelerometer cut-off points for calculating moderate-to-vigorous physical activity in young children. J Phys Act Health. 2007:4:4

17. Kim Y, Beets MW, Pate RR, Blair SN. The effect of reintegrating Actigraph accelerometer counts in preschool children: comparison using different epoch lengths. J Sci Med Sport. 2013;16:2.

18. Tucker $P$. The physical activity levels of preschool-aged children: a systematic review. Early Child Res Q. 2008;23:4.

19. Cooper AR, Goodman A, Page AS, Sherar LB, Esliger DW, van Sluijs EM, Andersen LB, Anderssen S, Cardon G, Davey R, et al. Objectively measured physical activity and sedentary time in youth: the international children's accelerometry database (ICAD). Int J Behav Nutr Phys Act. 2015;12:113.

20. Trost SG, Sirard JR, Dowda M, Pfeiffer KA, Pate RR. Physical activity in overweight and nonoverweight preschool children. Int J Obes Relat Metab Disord. 2003:27:7.

21. Messerli-Burgy N, Kakebeeke TH, Arhab A, Stulb K, Zysset AE, LeegerAschmann CS, Schmutz EA, Fares F, Meyer AH, Munsch S, et al. The Swiss Preschoolers' health study (SPLASHY): objectives and design of a prospective multi-site cohort study assessing psychological and physiological health in young children. BMC Pediatr. 2016;16:1.

22. Rolland-Cachera MF. Childhood obesity: current definitions and recommendations for their use. Int J Pediatr Obes. 2011;6:5-6.

23. Janssen X, Basterfield L, Parkinson KN, Pearce MS, Reilly JK, Adamson AJ, Reilly JJ. Gateshead millennium study Core T. objective measurement of sedentary behavior: impact of non-wear time rules on changes in sedentary time. BMC Public Health. 2015;15:504.

24. Hinkley T, O'Connell E, Okely AD, Crawford D, Hesketh K, Salmon J. Assessing volume of accelerometry data for reliability in preschool children. Med Sci Sports Exerc. 2012;44:12.

25. Penpraze $V$, Reilly JJ, MacLean CM, Montgomery C, Kelly LA, Paton JY, Aitchison T, Grant S. Monitoring of physical activity in young children: how much is enough? Pediatr Exerc Sci. 2006;18:4.

26. Pate RR, O'Neill JR. Physical activity guidelines for young children: an emerging consensus. Arch Pediatr Adolesc Med. 2012;166:12.

27. Department of Health PA, Health Improvement and Protection. Start Active, Stay Active: A Report on Physical Activity for Health from the Four Home Countries' Chief Medical Officers. In. London, England: Department of Health, Physical Activity, Health Improvement and Protection; 2011.

28. Department of Health and Ageing. National Physical Activity Guidelines for Australians: Physical Activity Recommendations for 0-5 Year Olds. In. Canberra, Australia: Commonwealth of Australia; 2010.

29. National Association for Sport and Physical Education. Active start: A statement of physical activity guidelines for children birth to 5 years. In. Oxon Hill, MD: AAHPERD Publications; 2002

30. Jimmy G, Seiler R, Maeder U. Development and validation of energy expenditure prediction models based on GT3X accelerometer data in 5to 9-year-old children. J Phys Act Health. 2013;10:7.

31. Banda JA, Haydel KF, Davila T, Desai M, Bryson S, Haskell WL, Matheson D, Robinson TN. Effects of varying epoch lengths, Wear time algorithms, and activity cut-points on estimates of child sedentary behavior and physical activity from accelerometer data. PLoS One. 2016;11:3.

32. Vale S, Santos R, Silva P, Soares-Miranda L, Mota J. Preschool children physical activity measurement: importance of epoch length choice. Pediatr Exerc Sci. 2009;21:4.

33. Keadle SK, Shiroma EJ, Freedson PS, Lee IM. Impact of accelerometer data processing decisions on the sample size, wear time and physical activity level of a large cohort study. BMC Public Health. 2014;14:1210.

34. Jimmy G, Seiler R, Mäder U. Development and validation of GT3X accelerometer cut-points in 5- to 9-year-old children based on indirect calorimetry measurements. Schweizerische Zeitschrift für Sportmedizin und Sporttraumatologie. 2013;61:4.

35. Cain KL, Sallis JF, Conway TL, Van Dyck D, Calhoon L. Using accelerometers in youth physical activity studies: a review of methods. J Phys Act Health. 2013;10:3.

36. Vanhelst J, Beghin L, Salleron J, Ruiz JR, Ortega FB, Ottevaere C, Molnar D, Kafatos A, Manios Y, Widhalm K, et al. Impact of the choice of threshold on physical activity patterns in free living conditions among adolescents measured using a uniaxial accelerometer: the HELENA study. J Sports Sci. 2014;32:2.

37. Bornstein DB, Beets MW, Byun W, Welk G, Bottai M, Dowda M, Pate R. Equating accelerometer estimates of moderate-to-vigorous physical activity: in search of the Rosetta Stone. J Sci Med Sport. 2011;14:5. 
38. Brazendale K, Beets MW, Bornstein DB, Moore JB, Pate RR, Weaver RG, Falck RS, Chandler JL, Andersen LB, Anderssen SA, et al. Equating accelerometer estimates among youth: the Rosetta Stone 2. J Sci Med Sport. 2016;19:3.

39. Rowlands AV. Moving forward with accelerometer-assessed physical activity: two strategies to ensure meaningful, interpretable, and comparable measures. Pediatr Exerc Sci. 2018:30:4.

40. Berenson GS, Srinivasan SR, Bao W, Newman WP 3rd, Tracy RE, Wattigney WA. Association between multiple cardiovascular risk factors and atherosclerosis in children and young adults. The Bogalusa heart study. N Engl J Med. 1998;338:23.

41. Andersen LB, Riddoch C, Kriemler S, Hills AP. Physical activity and cardiovascular risk factors in children. Brit I Sport Med. 2011;45:11.

42. Stone MR, Rowlands AV, Middlebrooke AR, Jawis MN, Eston RG. The pattern of physical activity in relation to health outcomes in boys. Int J Pediatr Obes. 2009;4:4.

Ready to submit your research? Choose BMC and benefit from:

- fast, convenient online submission

- thorough peer review by experienced researchers in your field

- rapid publication on acceptance

- support for research data, including large and complex data types

- gold Open Access which fosters wider collaboration and increased citations

- maximum visibility for your research: over $100 \mathrm{M}$ website views per year

At BMC, research is always in progress.

Learn more biomedcentral.com/submissions 